Marquette University

e-Publications@Marquette

\title{
Walker-Assisted Gait in Rehabilitation: A Study of Biomechanics and Instrumentation
}

Rebecca Ann Bachschmidt

Marquette University

Gerald F. Harris

Marquette University, gerald.harris@marquette.edu

Guy G. Simoneau

Marquette University, guy.simoneau@marquette.edu

Accepted version. IEEE Transactions on Neural Systems and Rehabilitation Engineering, Vol. 9, No. 1 (March 2001): 96-105. DOI: (C) 2001 Institute of Electrical and Electronics Engineers (IEEE). Used with permission. 


\title{
Walker-assisted Gait In Rehabilitation: A Study Of Biomechanics and Instrumentation
}

\author{
R.A. Bachschmidt \\ Department of Biomedical Engineering, Marquette University \\ Milwaukee, WI \\ G.F. Harris \\ Department of Biomedical Engineering, Marquette University \\ Milwaukee, WI \\ G.G. Simoneau \\ Department of Physical Therapy, Marquette University \\ Milwaukee, WI
}

\begin{abstract}
While walkers are commonly prescribed to improve patient stability and ambulatory ability, quantitative study of the biomechanical and functional requirements for effective walker use is limited. To date no one has addressed the changes in upper extremity kinetics that occur with the use of a standard walker, which was the objective of this study. A strain gauge-based walker instrumentation system was developed for the six degree-of-freedom measurement of resultant subject hand loads. The walker dynamometer was integrated with an upper extremity biomechanical model. Preliminary system data were collected for seven healthy, right-handed young adults following informed consent. Bilateral upper extremity kinematic data were acquired with a six camera Vicon motion analysis system using a Micro-VAX workstation. Internal joint moments at the wrist, elbow, and shoulder were determined in the three clinical planes using the


NOT THE PUBLISHED VERSION; this is the author's final, peer-reviewed manuscript. The published version may be accessed by following the link in the citation at the bottom of the page.

inverse dynamics method. The walker dynamometer system allowed characterization of upper extremity loading demands. Significantly differing upper extremity loading patterns were Identified for three walker usage methods. Complete description of upper extremity kinetics and kinematics during walker-assisted gait may provide insight into walker design parameters and rehabilitative strategies.

\section{SECTION I.}

\section{Introduction}

In 1990, there were over 1690000 noninstitutionalized walker users in the United States. ${ }^{1}$ About $85 \%$ were over the age of 65 years, with a mean age of 75 years. Although the walker is a relatively simple device which provides external mechanical support and reduces weight bearing on the lower limbs, its use is not without risk. Over a six-year period (from 1987 to 1992) over 118000 (mean = 19 714/year) accidents occurred which involved walker use and which required emergency room treatment. ${ }^{2}$ Females comprised over $75 \%$ of those injured and the most common injuries were hip fractures and upper body trauma. ${ }^{2}$

There have been some reports in the literature which have compared basic gait metrics (stride length, cadence, velocity) and user satisfaction with differing walker designs. ${ }^{3-4,5,6,7,8}$ Energy studies measuring the oxygen consumption and heart rate of subjects using both differing frame types and usage patterns have also been conducted.910,11,12 The sagittal plane upper extremity kinematics of walker-assisted gait have been reported.13,14 Only a few studies have quantitated the loads placed on a standard walker frame during the assisted gait cycle.15-16,17,18,19,20,21 However, these studies lacked the instrumentation necessary to fully describe upper extremity kinetics during walkerassisted gait.

Crosbie ${ }^{13,14}$ investigated the upper extremity motion of walker-assisted ambulation for ten healthy subjects. The author modeled walker-assisted gait, in the sagittal plane only, using both a theoretical kinematic model and by tracking upper and lower extremity landmarks with a video-film system. Both analyses demonstrated that assisted ambulation with a conventional "pick-up" type walker was an activity in which the segmental motion was determined and constrained by the rigid walker frame. The upper limbs moved symmetrically and together. The forward displacement of the frame determined the forward motion of the feet and the gait cycle consisted of discrete episodes of frame and lower limb movement. The author noted that the hip joint was moved in a mostly flexed 
position throughout the assisted gait cycle and that current design of standard walking frames prevents the development of a hip extensor pattern.

The group of Adrezin, Cordaro, Wang, et al. ${ }^{17-18,19}$ studied the interaction of weight borne by the walker and by the lower extremities throughout the gait cycle. A standard aluminum tube walker was instrumented with strain gauges on each of the four legs. Parameters that were measured included axial forces, anterior-posterior and medial-lateral bending loads at each of the four legs. Loads ranged from 89.0-222.4 N vertically, 2.2-17.8 $\mathrm{N}$ anterior/posterior (AP), and 1.1-11.6 N medial/lateral (ML) at each of the four legs. Their work demonstrated that walker loading patterns differed among an adult population $(n=12)$ with various gait abnormalities including: tibia-fibula fracture, amputation, hemiparesis, hemiplegia, and cerebral palsy. They suggested that since the patient population studied had a wide range of disabilities and differing walker usage patterns, a need exists for future research leading to better understanding of walkers and new walker designs which address the varying needs of patients.

The group of Deathe, Pardo, and Winter $15,16,20,21$ also measured adult walker reaction forces using strain gauges mounted on the legs of a standard walker. The algebraic sum of force records from each of the four legs provided the total walker ground reaction forces. The average peak total ground reaction forces measured for a population of 6 amputees were: 7\% $\pm 3 \%$ body weight (BW) AP, 3.9\% $1.7 \%$ BW ML, and $51.2 \% \pm 23.5 \%$ BW vertically. Vertical reaction forces measured at each leg were additionally used to calculate the horizontal position of the center-of-pressure and assess walker stability. Their study of adult amputees showed that the left and right walker legs were loaded unevenly during the assisted-gait cycle. Analysis of results obtained from a lower limb amputee revealed the patient to be least stable in forward rotation throughout the entire walker stance period. Peak risks were observed during initial walker loading, mid-stance, and just before walker lift-off. Their instrumented walker was also used to select appropriate walker heights, identify upper extremity strength requirements, document the onset of tremor associated with fatigue, quantify patient stability, and identify risk periods within the gait cycle for a population of amputees.

Instrumentation of the legs of a standard, "pick-up" type walker has been shown to provide useful objective documentation of patient/walker reaction loads, center-ofpressure, and estimation of patient stability. The systems described in the literature, however, were not designed to provide complete six degree-of-freedom load measurement. Quantification of left and right hand loads could not be made with these systems and, therefore, full kinetic analysis of upper body internal joint loads in the three clinical planes 
was not possible. This paper presents a walker dynamometer system and upper extremity biomechanical model used for the measurement of three-dimensional loads at the wrist, elbow, and shoulder. It is hoped that description of upper extremity kinetics may beneficially influence walker design parameters and gait retraining strategies.

The specific objectives of this study were: 1) to develop a walker dynamometer capable of providing bilateral measurement of the three-dimensional forces and moments applied by the hands to a walker frame, 2) to develop a bilateral upper body kinematic and kinetic model capable of quantitatively describing three dimensional walker-assisted gait, and 3) to characterize the upper extremity walker-assisted gait kinematics and kinetics of a population of healthy adults as a function of specified lower limb weight bearing $(0 \%, 10 \%$, and $50 \%$ body weight).

\section{SECTION II.}

\section{Methods}

\section{A. Subject Selection and Testing}

The recruited subjects were able-bodied, right-handed adults ( $\mu=27.9 \pm 7.5$ years, $74.8 \pm 24.4 \mathrm{~kg}, 174 \pm 6.4 \mathrm{~cm}$ height). A total of 7 subjects were studied, four males and three females. Inclusion criteria included: no prior lower extremity surgery, no history of stroke or other musculoskeletal pathology, no cardiovascular limitation, no prior use of a walker, right- handedness, and informed consent.

Bilateral joint kinematic data were acquired with a six-camera Vicon (Oxford, UK) motion analysis system using a Micro-VAX 3100 workstation (Maynard, MA). Reflective markers, about $2 \mathrm{~cm}$ in diameter, were attached directly to the skin over anatomical landmarks with hypoallergenic, double-sided tape. The bilateral motion data were automatically synchronized by the system to the loading data obtained with the instrumented walker. Data were sampled at a minimum frequency of 60 frames per second. The three-dimensional joint loads were normalized-forces with respect to subject body weight and moments with respect to subject body weight and arm length. These quantities were expressed in terms of the segment local coordinate system and represented the reaction of the proximal segment on the distal segment.

The instrumented walker was used with protected weight bearing patterns of the right lower limb (0\%, 10\%, and 50\% body weight). These loading patterns were chosen to 
reflect those typically used by patients recovering from pelvis, hip, femur, or tibia-fibula fractures and who are often prescribed walkers. ${ }^{22} \mathrm{~A}$ battery-powered, audio feedback system was employed to ensure adherence to a specified weight bearing level. The audio alarm included Force Sensing ResistorsTM (Interlink Electronics, Santa Barbara, CA.) placed under the first and fifth metatarsal heads and the heel. A $0 \mathrm{KHz}$ (no tone), $1 \mathrm{KHz}$, or $2 \mathrm{KHz}$ alarm sounded if the sensors were loaded within, below, or above threshold limits, respectively. The alarm was adjusted to a percentage of each subject's body weight.

Initially, the testing protocol was explained to the subject and informed consent was requested. The subjects were asked to wear paper shorts and a sleeveless shirt. External reflective markers were attached directly to the skin of the arms, legs, neck, and pelvis. Baseline gait data were gathered by having the subject walk without a walker on a $10 \mathrm{~m}$ walkway at a freely selected cadence and stride length. A minimum of three acceptable trials were recorded per side.

Next, the subject was instructed on the use of a walker. The walker was adjusted to an appropriate height such that the top of the walker was aligned with the ulnar styloid when the subject stood beside the frame with arms held loosely in a relaxed position. ${ }^{23,24}$ Using a noninstrumented, practice walker, the subject was taught a three-count, delayed, five-point gait. They lifted the standard walker, moved it forward about 0.20 to $0.30 \mathrm{~m}$ in front of their toes without overstretching, took two steps into the frame moving the involved right lower limb first, and then repeated the process. The audio alarm ensured that the involved right lower limb was loaded to the specified limits. The subjects were allowed to practice before data collection until the prescribed pattern was consistently observed and the subject reported a feeling of confidence.

Subjects were then asked to walk on the $10 \mathrm{~m}$ walkway at their own cadence using the instrumented walker. Subjects were not given verbal encouragement during the experiments. Weight bearing levels were randomly assigned by selecting a sample from a table of random numbers. ${ }^{25}$ Randomization provided that confounding effects such as fatigue, learning, or motivation were equally distributed between the three weight bearing levels. A minimum of three trials were conducted for all subjects for each of the testing conditions. An average of two steps were recorded per trial.

\section{B. Walker Dynamometer}

1) Finite Element Model: A walker is a statically indeterminate, three-dimensional space frame and it presents a more challenging and complex structural analysis problem 
than a cane or a crutch. Strain gauges mounted to the walker legs provide a total measure of load summed over the entire frame. To obtain a separate measurement of the load applied by each hand, an additional degree of static indeterminacy, "structural crosstalk," must be addressed. One aim of this study was to determine through finite element analysis the magnitude of load transmitted through the walker structural members when a load is applied to only one of the walker handles. Optimum strain gauge mounting locations were then specified for determination of forces and moments generated at each hand.

A standard, adult-sized, "pick-up" type walker frame was modeled using MARC finite element software (MARC Analysis Research Corporation, Palo Alto, CA). Translations of the legs along the ground were constrained. The finite element model was constructed using a straight three-dimensional beam element with six degrees-of-freedom, three rotations and three translations at each of the two connectivity points. The beam cross-section was a thin-walled circular cylinder with sixteen points of numerical integration. Stresses and strains were computed at each of the integration points throughout the cross section using Simpson's rule. Moments and total axial forces were integrated numerically along element axes using Gaussian integration. The first Gaussian integration point was near the first connectivity point, the second was at the beam midspan, and the third was near the second connectivity point. Numerical integration, or quadrature, was used to evaluate the complicated integrals. For example, formulation of a three-dimensional element stiffness matrix expressed in terms of local coordinates takes the form

$$
[k]=\int[B]^{T}[E][B] t J d \xi d \eta d \zeta(1)
$$

where

$[B] \quad$ strain-displacement relationship associated with the shape function and geometry of each element;

$[E] \quad$ stress-strain relationship;

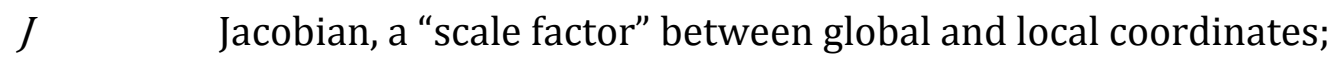

$\xi, \eta, \quad, \zeta$ local coordinates;

$t \quad$ element thickness.

The general quadrature formula approximates an integral, $I$, by evaluating the function $\Phi(\xi, \eta, \zeta)$ (a transformation of the original integrand by the Jacobian) at several locations, $\xi,, \eta,, \zeta$, and multiplying each by an appropriate weight, $W_{i}$, such that: 
NOT THE PUBLISHED VERSION; this is the author's final, peer-reviewed manuscript. The published version may be accessed by following the link in the citation at the bottom of the page.

$$
\begin{aligned}
& I=\iiint \Phi(\xi, \eta,, \zeta) d \xi d \eta d \zeta \\
& \cong \quad \Sigma \Sigma \Sigma W_{i} W_{j} W_{k} \Phi\left(\xi_{i}, \eta_{j}, \zeta_{k}\right) .
\end{aligned}
$$

Using this complex element with multiple integration points advantageously allowed fewer elements to be used in the model without encountering mesh instabilities.

Three orthogonal forces and moments were separately applied at a single node which represented one of the walker's handles. By applying loads at only one handle, the structural crosstalk in all frame members was fully described. Reaction moments were taken with respect to local axes about each element centroid. The displaced walker configuration and the fraction of the load carried by each member for the six loading cases were determined.

2) Walker Instrumentation: Resistive strain gauges are widely used in biomechanical load measurement systems and were chosen for implementation in the walker dynamometer system. All Micromeasurements EA series, $350-\Omega$ strain gauges were used (Measurements Group, Raleigh, NC). The gauges selected for the walker dynamometer were from 2.54 to $6.35 \mathrm{~mm}$ in length. An epoxy-type bonding agent was favored over cyanoacrylate for long-term stability.

A total of twelve unknowns, three forces and three moments applied by each hand, were to be determined. Twelve strain gauge bridges were arranged so that each experienced measurable strains under the action of one of the loads while remaining relatively insensitive to all others. A standard walker was instrumented with six sets of strain gauge bridges on each of the walker's two handle beams. Bending, Poisson, and $45^{\circ}$ rosette patterns were used (Fig. 1). The bridges were oriented such that upper extremity forces and moments could be resolved for analysis. Four strain gauge bridges sensitive to bending forces and moments were mounted fore and aft of each handle beam. Two of these four bridges measured bending loads in the transverse plane and two measured bending loads in the sagittal plane. This redundant gaging was necessary to uncouple forces and moments. A system of two equations of static equilibrium were solved for the two unknowns (one force and one moment) in the transverse and sagittal planes. Also, one bridge sensitive to axial torque and one tension/compression bridge was mounted on each handle. Redundant gaging was not necessary for these bridges because identical bending strains cancelled in opposite arms of the Wheatstone bridge. 


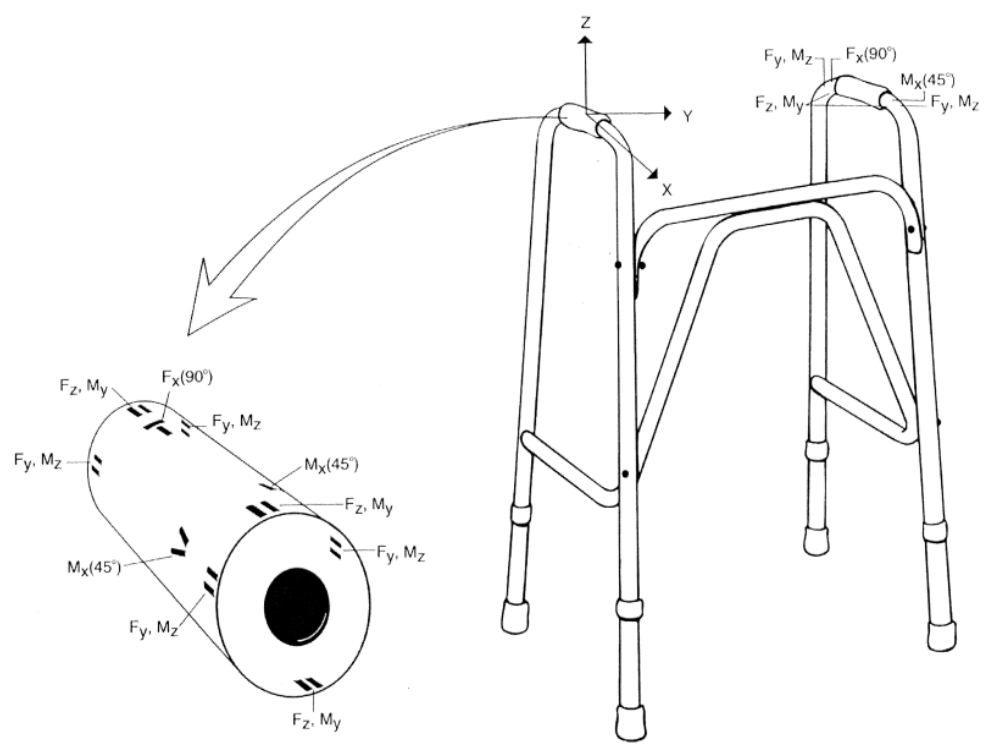

Fig. 1. Standard walker instrumented with twelve strain gauge bridges.

The strain gauge bridges were powered with $\mathrm{a} \pm 5-V$ dc source. Voltage signals from the strain gauge bridges were amplified with precision, micropower, single supply instrumentation amplifiers (Linear Technology LT1101, Milpitas, CA). Gains of 10000 were set for the Poisson and torque bridges; gains of 1000 were used for all other bridges. A 15 $\mathrm{Hz}$ anti-aliasing filter was included in the system instrumentation. The cutoff frequency was chosen above the documented frequency content of human gait. ${ }^{26}$ The walker dynamometer was tethered to a 12-bit A/D interface (Data Translation DT3382, Marlboro, MA) and computer workstation with 30 gauge, multistranded, spiral shielded cable.

Before each data collection session, the walker strain gauge system was powered for 30 minutes to allow instrumentation temperature stabilization. Static calibration of the load-sensing walker was continually verified prior to each test series using the six-axis floor-mounted force platforms (AMTI, OR6-5, Newton, MA).

3) Static Calibration: The walker dynamometer was statically calibrated using a multi-axis frame designed to apply known axial, bending, and torsional loads unilaterally. Standardized weights were repeatedly hung from cables and were supported by a pulley system designed to minimize frictional losses. Forces up to 10 pounds were incrementally applied to each of the walker handles in both the medial-lateral and anterior-posterior directions. The loading range was chosen to reflect the magnitude of similar forces reported in the literature. ${ }^{15,18}$ Forces ranging to 50 pounds were applied vertically to the walker handles. The upper loading limit was chosen so that frame stability was maintained during calibration. Torques were generated by applying a force at a known distance (2.54 
$\mathrm{cm}$ ) from the walker handle with correction for the strain gauge response due only to force loading.

Gauge sensitivity was determined by linearizing output voltages with respect to known applied loads. The system behavior was characterized by using the relationship ${ }^{27}$

$$
[V]=[S M][F](3)
$$

where $[V]$ is a column vector representing output voltages and $[F]$ is a column vector representing applied loads. The square sensitivity matrix [SM] relates applied loads to measured output. The sensitivity matrix was also written as follows:

$$
[S M]=[S]^{*}[K] \cdot(4)
$$

The $S_{i i}$ indicate the primary bridge sensitivities while the $K_{i j}$ include crosstalk components. The calibration matrix $[C]$ was determined as the inverse of the sensitivity matrix. Primary sensitivities were used in calculating the calibration matrix. The $[C]$ matrix components were then multiplied by output voltages to determine load values. The effect of mechanical crosstalk between the gauges mounted on the left and right sides of the walker frame was determined, as reflected in the $12 \times 12$ dimension of the sensitivity, crosstalk, and calibration matrices.

4) Dynamic Validation: The instrumented walker was dynamically validated for normal adult gait loading using a pair of floor-mounted AMTI force plates. The left side walker legs (front and back) contacted one force plate and the right side walker legs contacted the second force plate. Unilateral and bilateral dynamic hand loads were applied to the walker frame. Individual force components and total force magnitude measured with the walker were compared to that measured by the force plates. The walker was designed to sense the moments applied by the hands to the handles. The force plates, however, record an additional moment produced by the hand forces multiplied by the distance offset from force plate center. To compare the walker and force plate signals, the vector quantity of hand force (measured by the walker) crossed with the appropriate lever arm was subtracted from the force plate vector. 
NOT THE PUBLISHED VERSION; this is the author's final, peer-reviewed manuscript. The published version may be accessed by following the link in the citation at the bottom of the page.

\section{Upper Extremity Biomechanical Model}

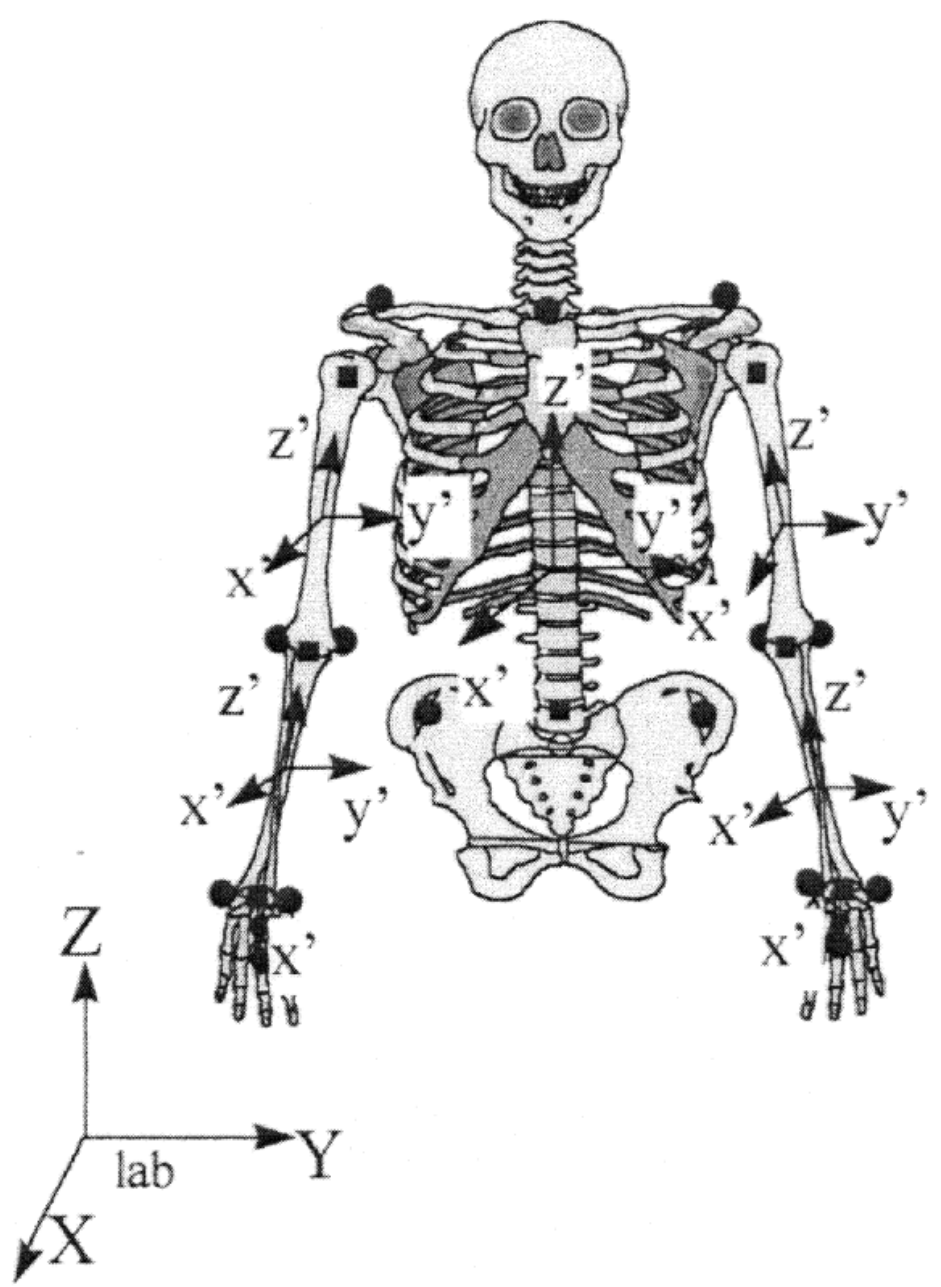

Fig. 2. Upper extremity marker locations and segment local coordinate systems.

The upper body segments tracked included: the trunk, upper arm, forearm, and hand. External reflective markers were attached to upper extremity anatomical landmarks. The marker coordinate data were used to determine the rotations of the upper body joints using an Euler angle approach, similar to that used by Kadaba, et al. [28].

The global coordinates of the markers (initially referenced with respect to the laboratory) were used to create orthogonal body coordinate systems which were directed from the distal segment toward the proximal segment. Typically, one unit vector was defined along the segment long axis. A second unit vector was established within a selected

IEEE Transactions on Neural Systems and Rehabilitation Engineering, Vol 9, No. 1 (March 2001): pg. 96-105. DOI. This article is (C) Institute of Electrical and Electronics Engineers (IEEE) and permission has been granted for this version to appear in e-Publications@Marquette. Institute of Electrical and Electronics Engineers (IEEE) does not grant permission for this article to be further copied/distributed or hosted elsewhere without the express permission from Institute of Electrical and Electronics Engineers (IEEE). 
plane defined by three points (marker and joint center locations) and was perpendicular to the first body segment unit vector. A third unit vector was constructed perpendicular to the other two unit vectors using vector cross products.

The trunk was defined by markers at the left and right anterior superior iliac spines (ASIS), vertebra C7, and sternal notch (Fig. 2). The pelvic center was defined as the midpoint between the LASIS and RASIS. The trunk length axis $(z)$ was then established as passing through the pelvic center to vertebra C7. The $x$-axis was defined perpendicular to the $z$-axis and within the plane of the suprasternal landmark. The $y$-axis was normal to both the $x$ - and $y$-axes.

The upper arm was defined by markers on the acromion process and the lateral and medial humeral epicondyles. The glenohumeral joint was represented as a perfect ball-andsocket joint with no translation of the rotation center of the humerus. Its rotation center was located using equations presented by Van Der Helm, Veeger et al.1992.29 The elbow joint center was assumed to lie halfway between the humeral epicondyles. The humerus long axis $(z)$ was then established as passing through the elbow center to the center of the humeral head. The $y$-axis was defined between the elbow center and epicondyles and was perpendicular to the $z$-axis. The $x$-axis was normal to both the $y$-and $z$-axes.

The forearm was modeled as one rigid link. The forearm was defined by the elbow center and by markers placed on the radial and ulnar styloids. The wrist joint center was assumed to lie halfway between the radial and ulnar styloids. The forearm long axis $(Z)$ was then established as passing through the wrist joint center to the center of the elbow center. The $y$-axis was defined between the wrist joint and styloids and was perpendicular to the $z$ axis. The $x$-axis was normal to both the $y$ - and $z$-axes.

Only two markers were required to describe the two angular motions of the hand, flexion/extension and radial/ulnar deviation. One unit vector was calculated from the line segment joining metacarpal III (MTP joint of the long finger) to the estimated wrist joint center. Marker coordinate data were low pass filtered using a $6 \mathrm{~Hz}$, dual-pass, second-order Butterworth, infinite impulse response (IIR) algorithm. ${ }^{30,31}$

The bilateral upper extremity motion data were synchronized to the loading data obtained with the force-sensing walker. By using the measured forces and moments applied to the walker in conjunction with detailed kinematic data of the upper extremities and anthropometric relationships, the loads borne by the upper body joints (Fig. 3) were calculated using inverse dynamics. ${ }^{32}$ 


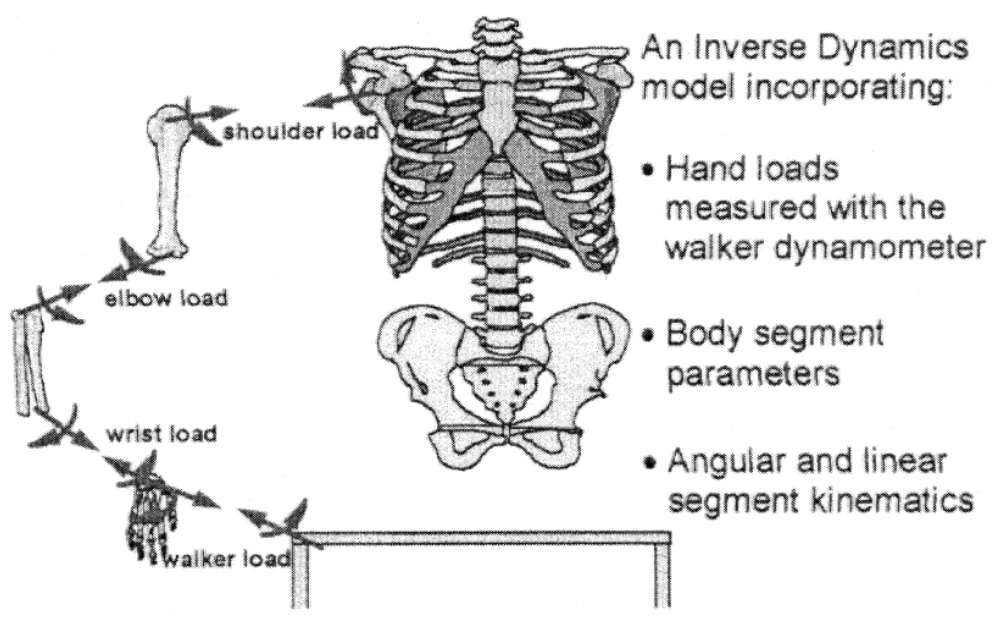

Fig. 3. Upper extremity kinetic model.

Segmental masses, centers-of-mass, and mass moments of inertia were estimated using anthropometric relationships. Masses were estimated as percentages of total body mass with units of kilograms. ${ }^{30}$ Centers-of-mass were estimated using the equations of Chandler, $1975 .{ }^{33} \mathrm{COM}$ locations were calculated with respect to laboratory global coordinates. Mass moments of inertia $\left(I_{i i}\right)$ were estimated using the dimensionally consistent, nonlinear regression equations of Yeadon and Morlock, 1989.34 The segment reference axes were assumed to coincide with the principle axes of inertia and the products of inertia were assumed to be equal to zero.

\section{Data Analysis}

The measured variables included temporal and spatial parameters (cadence, velocity, stride length, and stance-to-swing ratios), kinematic variables (joint angular positions, velocities, and accelerations), and kinetic variables (walker loads, internal joint forces and moments). The walker-assisted stride cycle was defined from walker contact to subsequent walker contact and included the following events: 1) walker contact, 2) foot off, 3) foot contact 4) opposite foot off, 5) opposite foot contact, 6) walker lift off, and 7) walker contact. ${ }^{15}$ Walker stance was defined as the period from initial walker contact to walker lift off and walker swing was defined from walker lift off to second walker contact.

Data were time normalized as a percentage of the walker stride cycle. Average and standard deviation values were calculated across three bilateral strides for each subject for each testing condition ( $0 \%, 10 \%, 50 \%$ body weight). Maximum and minimum walker stance phase variables were identified from each subject's stride $(n=3$ bilateral 
strides/subject, $N=7$ subjects). Differences among the variable maximums and minimums for the three weight bearing levels were investigated using the Mann-Whitney U test and the Wilcoxon Matched Pairs test. Nonparametric statistics, which do not assume a normal population distribution, were used due to the small sample size. The null hypothesis was that the mean gait characteristics were equivalent across the three walking conditions. The decision rule was to reject the null hypothesis if it was significant at the $5 \%$ level.

TABLE I Transducer Primary Sensitivities

\begin{tabular}{c|c|c|c|c|c}
\hline \multicolumn{3}{c|}{ Forces (mV/N) } & \multicolumn{3}{|c}{ Moments (mV/Ncm) } \\
$\begin{array}{c}\text { Fx } \\
\text { (A/P) }\end{array}$ & $\begin{array}{c}\text { Fy } \\
(\mathrm{M} / \mathrm{L})\end{array}$ & $\begin{array}{c}\mathrm{Fz} \\
\text { (vertical) }\end{array}$ & $\begin{array}{c}\text { Mx } \\
\text { (coronal) }\end{array}$ & $\begin{array}{c}\text { My } \\
\text { (sagittal) }\end{array}$ & $\begin{array}{c}\mathrm{Mz} \\
\text { (transverse) }\end{array}$ \\
\hline 123.55 & 35.10 & 3.80 & 0.40 & 3.95 & 0.85 \\
\hline
\end{tabular}

TABLE II Crosstalk Characterization

\begin{tabular}{c|c|c|c}
\hline Cross-talk & Sagittal Plane & Coronal Plane & Transverse Plane \\
\hline mean(sd) & $0.20(0.26)$ & $0.11(0.16)$ & $0.17(0.24)$ \\
median & 0.07 & 0.05 & 0.06 \\
mode & 0.02 & 0.05 & 0.05 \\
range & $0.00-0.84$ & $0.00-0.68$ & $0.00-0.84$ \\
\hline
\end{tabular}

\section{SECTION III.}

\section{Results}

1) Finite Element Analysis: For a vertical force applied downward on one handle, about $22 \%$ of the maximum axial force was carried in the opposite front leg. Local bending moments and axial torque were as great as $40 \%$ of maximum in the lower legs on the contralateral side. Less than $10 \%$ of the largest loads, both forces and moments, was noted in the opposing handle.

When shear forces were applied to a handle, the lower legs on the opposite side bore a greater fraction of force than did the lower legs on the side of loading. For a medially directed shear force, loads transmitted to the contralateral legs were as great as $46 \%$ and $83 \%$, for axial force and local bending moments, respectively. Again, loads experienced in the opposite handle were smaller, ranging from 3 to $12 \%$. For an anteriorly directed shear 
force, the contralateral legs bore about $55 \%$ of the axial load and experienced the greatest, $100 \%$, local bending moment. The opposite handle registered $7 \%$ of the maximum load.

A large degree of structural crosstalk occurred for moments applied about the handle's vertical axis. For this loading condition, the contralateral legs experienced the largest magnitude of axial load, $100 \%$, and $48 \%$ bending moment. Normalized axial load at the handle was $23 \%$ and normalized moments were all less than $4 \%$.

Finite element analysis demonstrated that the legs of the walker were the members most greatly affected by structural crosstalk. In contrast, the handle beams of the walker were more structurally isolated than were the legs. The handle beams were therefore chosen as the preferred gaging locations for measurement of hand loads with a minimum of structural crosstalk.

2) Sensor Characterization: The experimentally determined primary sensitivities in units of $\mathrm{mV} / \mathrm{N}(\mathrm{cm})$ are shown in Table I. Sensitivities were maximum in the anteriorposterior Fx loading direction and minimum in the vertical Fz loading direction. The experimentally determined crosstalk coefficients recorded for all loading conditions (144 elements) are characterized in Table II. Percentage crosstalk contralateral to the side of load application averaged 7.3\%. Inclusion of crosstalk coefficients in the output computations greatly decreased the errors due to crosstalk.

TABLE III Subject Time-DistanceParameters (Mean Cadence, Velocity, and Stride Length). N=7 Subjects, $\mathrm{n}=42$ Walker Stride Cycles PerLower Extremity Weight Bearing Level

\begin{tabular}{c|c|c|c|c|c|c|c|c|c}
\hline & \multicolumn{3}{|c|}{ cadence (step/min) } & \multicolumn{3}{c}{ velocity (m/s) } & \multicolumn{3}{c}{ stride length (m) } \\
\hline $\begin{array}{c}\text { lower } \\
\text { extremity } \\
\text { weight } \\
\text { level }\end{array}$ & $0 \% \mathrm{BW}$ & $\mathbf{1 0 \%} \mathrm{BW}$ & $\mathbf{5 0 \%} \mathrm{BW}$ & $0 \% \mathrm{BW}$ & $10 \% \mathrm{BW}$ & $\mathbf{5 0 \% \mathrm { BW }}$ & $0 \% \mathrm{BW}$ & $\mathbf{1 0 \% \mathrm { BW }}$ & $\mathbf{5 0 \%} \mathrm{BW}$ \\
\hline mean & 54.3 & 44.8 & 38.9 & 0.29 & 0.22 & 0.17 & 0.63 & 0.61 & 0.55 \\
\hline std dev & 13.4 & 13.1 & 8.9 & 0.07 & 0.07 & 0.04 & 0.06 & 0.17 & 0.18 \\
\hline
\end{tabular}

Gauges loaded in the primary design directions displayed maximum nonlinearities $\leq 3.0 \%$ of full scale output for increased load at any data point. The nonlinearities of the crosstalk signals were greater than those of the primary signals. In the gauge nondesign directions nonlinearities of 3-20\% were recorded. The greater nonlinearities were noted for the bridges contralateral to the side of load application. Sums-of-square-error computed for the linear regression fit were on the order of $10^{-4}-10^{-5}$ for bending and Poisson patterns ipsilateral to load application. Increased sums-of-square-error, $10^{-2}-10^{-3}$, were determined for the torque bridges and for bridges contralateral to load. The 
calibration curves displayed minimal hysteresis, $2 \%$ to $4 \%$, as was expected for strain gauges mounted onto the linearly elastic metal. Peak signal to root-mean-square (rms) noise averaged $50 \mathrm{~dB}$ for all bridges.

It is recommended that future designs incorporate transducer elements micromachined to further optimize sensor characteristics. Transducer geometry can be machined to increase primary sensitivities, decrease crosstalk sensitivities, and establish strain fields which are both symmetrical about the neutral axes and independent of the points of load application. Inclusion of mathematical error functions describing nonlinear crosstalk may further minimize the effects of crosstalk.

3) Precision, Accuracy, Reliability: Precision in the gauge design directions ranged from $92 \%$ to $98 \%$, as determined from repeated static calibration. Accuracies, assessed from repeated dynamic validation using two floor-mounted AMTI force plates, were

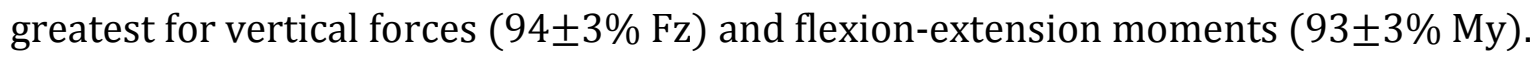
These were the largest magnitude loads noted for normal adult loading of the walker. Accuracies decreased for the remaining loads: $82 \pm 3 \% \mathrm{Fx}, 85 \pm 6 \% \mathrm{Fy}, 85 \pm 7 \% \mathrm{Mx}$, and $79 \pm 4 \% \mathrm{Mz}$. During a four month testing period, $90 \%$ of the walker dynamic validations were within one standard deviation of the stated mean accuracies for all loading directions. The system was durable over the testing period; no gauges or lead wires required repair or replacement over the testing.

4) Gait Metrics: In Table III, cadence decreased from 54.3 steps/min, to 44.8 steps/min and to 38.9 steps/min for the $0 \%, 10 \%$, and $50 \%$ body weight bearing patterns of the right leg, respectively. Decreasing velocities and stride lengths were also noted. Stance phase was prolonged on the leg on which weight bearing was not restricted. Stance to swing times were more equally distributed between the left and right legs for the $50 \%$ weight level than for the $10 \%$ weight level. The median walker stance time (initial walker contact to walker lift off) decreased in a linear fashion from about $57 \%$, to $44 \%$, and to $38 \%$ for the $50 \%, 10 \%$, and $0 \%$ weight levels, respectively.

5) Upper Extremity Kinematics: Fig. 4 illustrates the mean upper extremity joint angles bracketed by one standard deviation for a typical subject. The means were computed over at least three steps for each subject. For the study group, shoulder flexion was noted at initial walker contact in the sagittal plane. Shoulder flexion diminished and an extension pattern was seen as the subjects stepped into the frame. The shoulder was again flexed during walker swing as the subjects lifted the frame and moved it forward. Elbow flexion was a minimum at initial walker contact and increased as the subjects stepped into 
and loaded the frame. Maximum elbow flexion was obtained at initial walker swing. The wrist was maintained in an extended pattern with maximum extension noted during walker stance and frame loading.
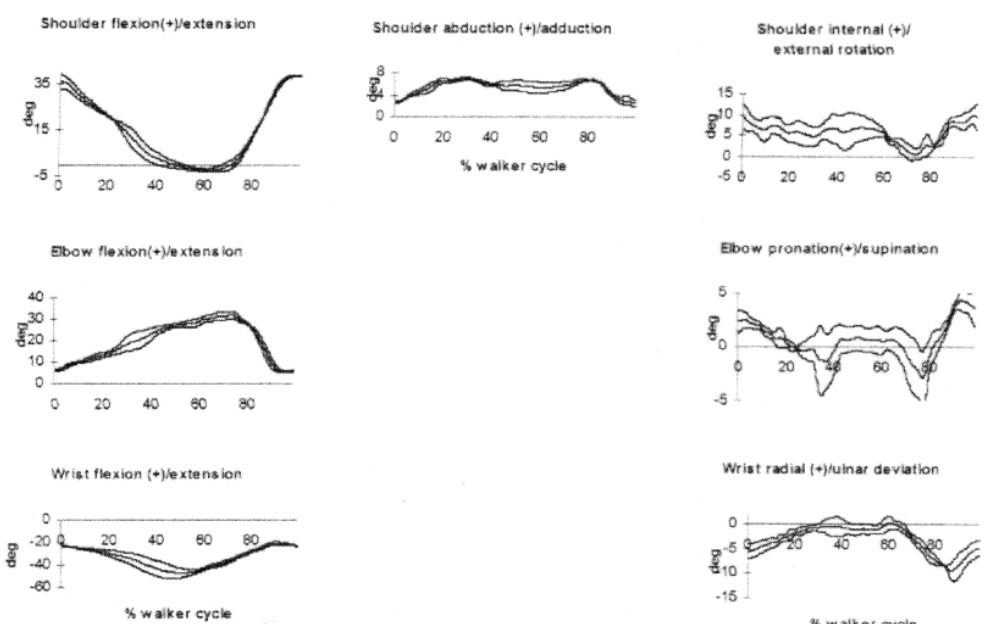

Fig. 4. Mean right upper extremity joint angles bracketed by one standard deviation of a typical subject for walker-assisted gait with $50 \%$ weight bearing on the right leg.

Coronal plane shoulder abduction was noted throughout the assisted-cycle and was greatest during early walker stance and early walker swing. Coronal plane motion was not determined for the elbow or the wrist.

In the transverse plane, the shoulder was internally rotated during walker stance. Maximum forearm pronation was noted during mid to late walker stance. The wrist was maintained in ulnar deviation during early walker stance and swing and was reduced during mid to late walker stance.

6) Upper Extremity Kinetics: Fig. 5 illustrates the mean upper extremity internal joint moments bracketed by one standard deviation for a typical subject. The means were computed over at least three steps for each subject. The moments, computed in units of $N * m$, were normalized with respect to subject body mass $(\mathrm{kg})$ and arm length $(\mathrm{m})$. 


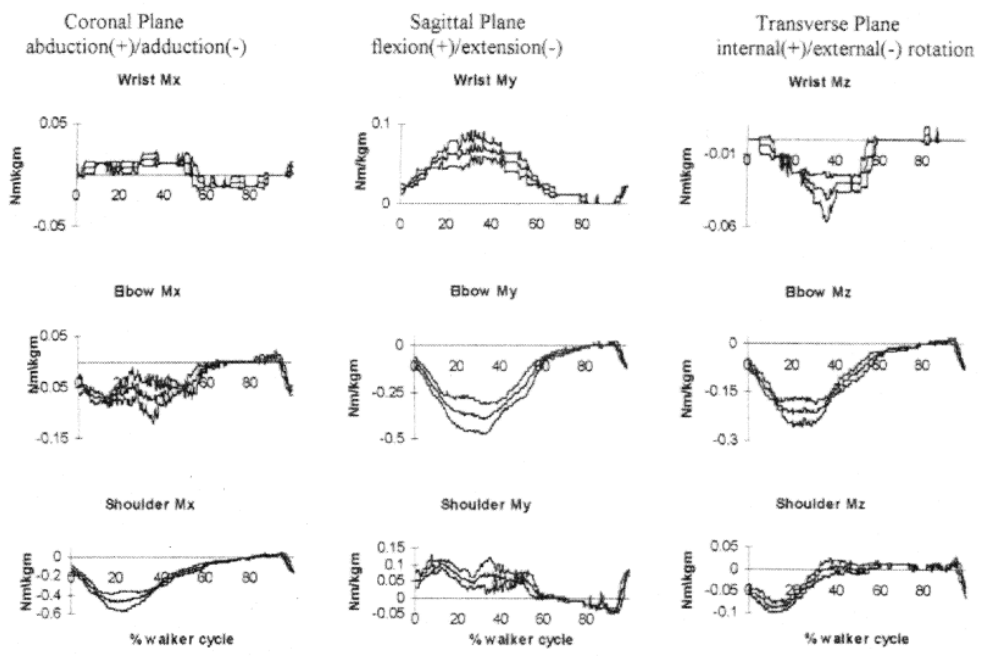

Fig. 5. Mean right upper extremity joint moments bracketed by one standard deviation of a typical subject for walker-assisted gait with $50 \%$ weight bearing on the right leg. (Wrist radial(+)/ulnar demands are reflected in the transverse plane, wrist Mz, as only one unit vector, $i$, was calculated along the hand long axis).

For the study group, demands on the shoulder flexors, elbow extensors, and wrist flexors were noted during walker stance in the sagittal plane. The greatest sagittal moment demands were recorded at the elbow ( $p \leq 0.05)$. In the coronal plane, adductor demands were noted at the shoulders and elbows with the shoulder demand being significantly greater than that at the elbow $(p \leq 0.05)$. Smaller magnitude radial demands were recorded at the wrists. In the transverse plane, demands were on the shoulder external rotators, elbow supinators, and wrist supinators. The greatest transverse moment demands were recorded at the elbow $(p \leq 0.05)$.

\section{SECTION IV.}

\section{Discussion}

The greatest ranges of upper extremity joint motion occurred in the sagittal plane, as anticipated. The maximum extension moment at the elbow during walker stance was significantly greater than moments at either the wrist or shoulder for all trials. The elbow joint was displaced into flexion with loading and an extensor moment would be necessary to support the upper body. Interestingly, a shoulder flexor demand was also noted. Perhaps this represents an eccentric demand on the shoulder flexors to control deceleration and the rate at which the walker frame is loaded. 
Coronal and transverse plane motions were also noted, especially at the shoulder. The shoulder was abducted and internally rotated as the frame was loaded. The maximum adduction moment at the shoulder during walker stance was significantly greater than moments at either the wrist or elbow. The demand on the shoulder adductors corresponds to the observed shoulder abduction which would tend to increase the moment arm from the distal to the proximal joint centers amplifying the effect of distal joint forces. In the transverse plane the maximum demand on external rotators during walker stance was at the elbow.

The peak magnitudes of internal joint moments noted during walker stance suggest that walker-assisted gait is an activity which is demanding to the upper body musculature. The greatest mean moment, about $37.3 \pm 11.7 \mathrm{~nm}$, corresponded to an elbow extensor demand for the $0 \%$ BW LE weight bearing condition. For comparison, it is reported in the literature that for normal level walking the sagittal plane peak knee extensor moment is 31.5-43.4 $\mathrm{nm}$ and the peak hip extensor moment is $42.0-84.0 \mathrm{~nm} .{ }^{35}$ Peak loads of about 42.0 and $21.0 \mathrm{~nm}$ have been reported for shoulder adductors and elbow extensors during reciprocal crutch aided gait in paraplegics. ${ }^{36}$ Peak loads of about $35.0 \mathrm{~nm}$ have also been reported for shoulder flexors and elbow extensors during swing-through crutch gait by paraplegics and able-bodied persons. ${ }^{37}$ In wheelchair users, average peak shoulder, elbow, and wrist moments of 19.6, 12.3 , and $5.8 \mathrm{~nm}$, respectively, have been reported. ${ }^{38}$

In addition to percentage lower limb loading, other parameters which could have affected both the upper body kinematics and kinetics include: walker height, upper limb length, subject weight, forward placement of walker, cadence, velocity, and stride length. The walker height was adjusted in the clinically accepted method of aligning the top of the frame with the ulnar styloid as the subject stands beside the frame with arms held loosely in a relaxed position. This method has been shown to provide between 20-30 degrees of elbow flexion in about $94 \%$ of normal subjects. ${ }^{24}$ Differences in subject arm lengths and body weights were accounted for by normalizing the moments with respect to these quantities. Forward placement of the walker, cadence, and stride length were not controlled. In lower extremity gait analysis, freely selected cadence and stride length is known to be consistent between individuals and directly related to the optimal efficiency for an individual. ${ }^{39}$ This was presumed to also be the case for walker-assisted gait. However, the changes in subject velocities with prescribed lower limb weight bearing patterns $(0 \%, 10 \%$, and $50 \%$ body weight) may be a source of variability in the joint kinetic data. Care must be taken when extrapolating the results of this sample group to the general population of walker users. The mean age of the study group was 27.9 years while the mean age of persons who use walkers is 75 years. ${ }^{40}$ The younger study group most likely 
exhibited faster walking speeds and generated larger moments of force than would an older study group.

Our preliminary work suggests that with a pick-up walker the arms partially take on the role of legs, supporting the body against ground reaction loads and experiencing intersegmental demands similar in magnitude to level walking. Potential rehabilitation applications which could benefit from this technology may include the study of pediatric walker-assisted gait. Reduction of upper extremity joint moment demands and improvement of efficiency remain as biomechanical design goals.

\section{References}

1Statistical Abstract United States 1993, DC, Washington:, vol. 8, pp. 140, 1993.

2P. M. Charron, R. L. Kirby, D. A. MacLeod, "Epidemiology of walker-related injuries and deaths in the United States", Amer. J. Phys. Med. Rehab., vol. 74, no. 3, pp. 235-239, 1995.

3"AARP Product report: Walkers", Amer. Assoc. Retired Persons, vol. 1, no. 7, pp. 1-15, 1991.

4J. Mahoney, R. Euhardy, M. Carnes, "A comparison of a two-wheeled walker and a three-wheeled walker in a geriatric population", J. Amer. Geriatrics Soc., vol. 40, pp. 208-212, 1992.

${ }^{5}$ G. L. Smidt, M. A. Mommens, "System of reporting and comparing influence of ambulatory aids on gait", Phys. Ther., vol. 60, no. 5, pp. 551-558, 1980.

${ }^{6}$ B. M. Greiner, J. M. Czernieck, J. C. Deitz, "Gait parameters of children with spastic diplegia: A comparison of effects of posterior and anterior walkers", Arch. Phys. Med. Rehab., vol. 74, pp. 381-385, 1993.

7P. K. Levangie, M. D. Chimera, M. L. Johnston, "The effects of posterior rolling walkers versus the standard rolling walker on gait characteristics of children with spastic cerebral palsy", Phys. Occup. Ther. Pediatr., vol. 9, pp. 1-17, 1989.

${ }^{8}$ L. Logan, K. Byers-Hinkley, C. Ciccone, "Anterior versus posterior walkers: A gait analysis study", Dev. Med. Child. Neurol., vol. 32, pp. 1044-1048, 1990.

${ }^{9}$ M. A. Hamzeh, P. Bowker, A. Sayegh, "The energy costs of ambulation using two types of walking frames", Clin. Rehab., vol. 2, pp. 119-123, 1988.

${ }^{10}$ C. G. Holder, E. M. Haskvitz, A. Weltman, "The effects of assistive devices on the oxygen cost cardiovascular stress and perception of nonweight-bearing ambulation", J. Orthop. Sports Phys. Ther., vol. 18, no. 4, pp. 537-542, 1993.

11L. J. Cordery, A. B. Ford, M. T. Ferrer, "Energy expenditure in assisted ambulation", J. Chronic Dis., vol. 7, no. 3, pp. 228-233, 1958.

12I. M. Baruch, K. A. Mossberg, "Heart-rate response of elderly women to nonweight bearing ambulation with a walker", Phys. Ther., vol. 63, pp. 1782-1787, 1983.

13J. Crosbie, "Kinematics of walking frame ambulation", Clin. Biomech., vol. 8, pp. 31-36, 1993.

14J. Crosbie, "Comparative kinematics of two walking frame gaits", J. Orthop. Sports Phys. Ther., vol. 20, no. 4, pp. 186-192, 1994. 
${ }^{15 R}$. D. Pardo, D. A. Winter, A. B. Deathe, "System for routine assessment of walker-assisted gait", Clin. Biomech., vol. 8, pp. 73-80, 1993.

16R. D. Pardo, A. B. Deathe, D. A. Winter, "Walker user risk index", Amer. J. Phys. Med. Rehab., vol. 72, no. 5, pp. 301-305, 1993.

17R. S. Adrezin, M. A. Cordaro, F. S. Wang, A. Fast, "Instrumentation and computer interfacing of a standard walker to study user-walker interaction dynamics", Adv. Bioeng. ASME, vol. 22, pp. 403-405, 1992.

${ }^{18}$ A. Fast, F. S. Wang, R. S. Adrezin, M. S. Cordaro, J. Ramis, J. Sosner, "The instrumented walker: Usage patterns and forces", Arch. Phys. Med. Rehab., vol. 76, pp. 484-491, 1995.

${ }^{19}$ R. S. Adrezin, M. A. Cordaro, F. S. Wang, A. Fast, J. Ramis, "A finite element model of a walker and its comparison to an instrumented walker", Adv. Bioeng. ASME, vol. 26, pp. 583-586, 1993.

${ }^{20}$ A. B. Deathe, R. D. Pardo, D. A. Winter, "Stability of walking frames", J. Rehab. Res. Dev., vol. 33, no. 1, pp. 30-35, 1996.

${ }^{21}$ A. B. Deathe, K. C. Hayes, D. A. Winter, "The biomechanics of canes crutches and walkers", Crit. Rev. Phys. Rehab. Med., vol. 5, no. 1, pp. 15-29, 1993.

22A. P. Ruskin, Current Therapy in Physiatry, PA, Philadelphia:WB Saunders, pp. 299-304, 1984.

${ }^{23}$ S. A. Nabizadeh, T. B. Hardee, M. A. Towler, V. T. Chen, R. F. Edlich, "Technical considerations in the selection and performance of walkers", J. Burn Care Rehab., vol. 14, no. 2, pp. 182-188, 1993.

${ }^{24 R}$. Kumar, M. C. Roe, O. U. Scremin, "Methods for estimating the proper length of a cane", Arch. Phys. Med. Rehab., vol. 76, pp. 1173-1175, 1995.

${ }^{25}$ S. B. Hulley, S. R. Cummings, "Designing a new study: Experiments" in Designing Clinical Research, MD, Baltimore:Williams and Wilkins, pp. 111-124, 1988.

${ }^{26}$ E. K. Antonsson, R. W. Mann, "The frequency content of gait", J. Biomech., vol. 18, no. 1, pp. 39-47, 985.

${ }^{27}$ G. F. Harris, E. A. Millar, "Lower extremity hypertonicity assessment: A computer-based system", J. Clin. Eng., pp. 453-458, Nov./Dec. 1990.

${ }^{28}$ M. P. Kadaba, H. K. Ramakrishnan, M. E. Wootten, "Measurement of lower extremity kinematics during level walking", J. Orthop. Res., vol. 8, pp. 383-392, 1990.

${ }^{29}$ F. C. T. Van Der Helm, H. E. J. Veeger, G. M. Pronk, L. H. V. Van Der Woude, R. H. Rozendal, "Geometry parameters for musculoskeletal modeling of the shoulder system", J. Biomech., vol. 25, no. 2, pp. 129-144, 1992.

${ }^{30}$ D. A. Winter, Biomechanics and Motor Control of Human Movement, New York:Wiley, 1990.

${ }^{31}$ D. A. Winter, H. G. Sidwall, D. A. Hobson, "Measurement and reduction of noise in kinematics of locomotion", J. Biomech., vol. 7, pp. 157-159, 1974.

${ }^{32}$ C. L. Vaughan, B. L. Davis, J. C. O'Connor, Gait Anal. Lab. Gaitmath, IL, Champaign:Human Kinetics, pp. 83-106, 1992.

${ }^{33} \mathrm{~F}$. R. Chandler, C. E. Clauser, J. T. McConville, H. M. Reynolds, J. W. Young, Investigation of inertial properties of the human body, Wright Patterson Air Force Base, pp. 69-70, 1975.

${ }^{34}$ M. R. Yeadon, M. Morlock, "The appropriate use of regression equations for the estimation of segmental inertia parameters", J. Biomech., vol. 22, pp. 683-689, 1989.

IEEE Transactions on Neural Systems and Rehabilitation Engineering, Vol 9, No. 1 (March 2001): pg. 96-105. DOI. This article is @ Institute of Electrical and Electronics Engineers (IEEE) and permission has been granted for this version to appear in e-Publications@Marquette. Institute of Electrical and Electronics Engineers (IEEE) does not grant permission for this article to be further copied/distributed or hosted elsewhere without the express permission from Institute of Electrical and Electronics Engineers (IEEE). 
${ }^{35}$ G. F. Harris, P. A. Smith, "Three-dimensional moments power and work in normal gait: Implications for clinical assessment" in Human Motion Analysis Current Applications and Future Directions, NJ, Piscataway:IEEE Press, pp. 74-77, 1996.

36W. J. Crosbie, A. C. Nicol, "Reciprocal aided gait in paraplegia", Paraplegia, pp. 353-363, 1990.

${ }^{37}$ L. Noreau, C. L. Richards, F. Comeau, D. Tardif, "Biomechanical analysis of swing-through gait in paraplegic and nondisabled individuals", J. Biomech., vol. 28, pp. 689-700, 1995.

38R. N. Robertson, M. L. Boninger, R. A. Cooper, S. D. Shimada, "Pushrim forces and joint kinetics during wheelchair propulsion", Arch. Phys. Med. Rehab., vol. 77, pp. 856-864, 1996.

39J. Rose, J. G. Gamble, Human Walking, MD, Baltimore:Williams and Wilkins, pp. 25, 1994.

${ }^{40} \mathrm{G}$. Mulley, "Everyday aids and appliances walking frames", BMJ, vol. 300, pp. 925-927, 1990. 